\title{
Silicon oxides as alignment surfaces for vertically-aligned nematics in photonic devices
}

\author{
E. OTON ，S. LÓPEZ-ANDRÉS , N. BENNIS , J.M. OTÓN , M.A. GEDAY
}

A comparative study on alignment performance and microstructure of inorganic layers used for liquid crystal cell conditioning has been carried out. The study has focused on two specific materials, SiOx and $\mathrm{SiO}_{2}$, deposited under different conditions. The purpose was to establish a relationship between layer microstructure and liquid crystal alignment. The surface morphology has been studied by FESEM and AFM. An analysis on liquid crystal alignment, pretilt angle, response time, contrast ratio and the conditions to develop backflow effect (significant rise time increase due to pure homeotropic alignment) on vertically-aligned nematic cells has been carried out. A technique to overcome the presence of backflow has been identified. The full comparative study of $\mathrm{SiOx}$ and $\mathrm{SiO}_{2}$ layer properties and their influence over liquid crystal alignment and electrooptic response is presented.

Keywords: $\mathrm{SiOx}, \mathrm{SiO}_{2}$, silicon oxides, vertically aligned nematics, photonic devices, morphology characterization.

\section{Introduction}

Inorganic layers are commonly used in alignment of liquid crystal devices, either parallel to the glass plates (homogeneous alignment) or perpendicular to the plates (homeotropic alignment, vertical alignment) [1]. However, the layer microstructure and its influence on the liquid crystal alignment is not fully understood yet. Performance of liquid crystal in a cell depends on several factors, including interaction between the liquid crystal monolayer next to the substrates and the alignment layer [2,3]. These interactions determine the liquid crystal pretilt and polar anchoring energy of the cell. This affects crucial electrooptic factors $[4,5]$, such as threshold voltage and response time of the device [6,7], which may be modified by varying different manufacturing process parameters [8-11].

The study was focused on $\mathrm{SiOx}$ and $\mathrm{SiO}_{2}$ layers deposited by thermal or e-gun evaporation. The alignment and microstructure of inorganic layers used for liquid crystal cell conditioning when the oxides are deposited under different conditions was studied to find a relationship between layer microstructure and liquid crystal alignment. Employing advanced microscopy techniques, the study of SiOx and $\mathrm{SiO}_{2}$ surfaces has proven useful to understand how surface morphology is modified and the influence on alignment in vertically-aligned nematics (VANs), along with their response time, threshold voltage and contrast ratio.

\section{Experimental}

A comparison between $\mathrm{SiOx}$ and $\mathrm{SiO}_{2}$ layer surface characteristics has been carried out. The evaporation parameter to be modified on each set of samples was the angle of incidence of the material being deposited onto the substrates. The comparative study was divided into the following steps:

- Observing surface morphologies variation by microscopy;

- Manufacturing test cells with VANs for the different surface structures;

- Finding out a relationship between surface morphology and the way the liquid crystal homeotropic alignment is modified;

- Studying electrooptic properties of the manufactured cells and establishing a manufacturing protocol.

Both $\mathrm{SiOx}$ and $\mathrm{SiO}_{2}$ depositions are carried out in the evaporation system, employing thermal evaporation or e-gun, respectively. The evaporation system is a modified Vacudel 300 Telstar equipment.

Deposition rate was fixed to $1 \AA / s$ in all the alignment layers. The reason for choosing this speed is to ensure a correct deposition of the material for the structure to grow on glass substrates adequately. Layer thickness was set to $100 \AA$ for all samples.

Layer thickness (d) is determined by the evaporation program with layer parameters, quartz sensor parameters and material parameters using the following equation 


$$
d=\left(\frac{\varpi \rho_{\text {quartz }}}{\pi \rho_{\text {layer }} Z \Phi_{\text {sensor }}}\right) \arctan \left\{Z \tan \left[\frac{\pi\left(\Phi_{l s}-\Phi_{\text {sensor }}\right)}{\varpi_{l s}}\right]\right\}
$$

Where:

$\varpi \quad$ is the frequency constant $1.668 \cdot 10^{13} \mathrm{~Hz} \cdot \AA$,

$\varpi_{\text {sensor }}$ is the quartz crystal frequency before the evaporation process,

$\varpi_{l s} \quad$ is the quartz crystal frequency after the evaporation process,

$\rho_{\text {quarz }}$ is the quartz crystal density,

$\rho_{\text {laver }}$ is the evaporated material density,

$\mathrm{Z}=\mathrm{Z}$ factor.

$\mathrm{Z}$ factor is a typical term tabulated for each material. It is introduced as a parameter in the evaporation program so the final thickness is correctly determined. $\mathrm{Z}$ factor depends on quartz density and material to be deposited.

The first aim was to find out the evaporation angle range that induces liquid crystal alignment variations. A series of samples was manufactured varying evaporation angle from $0^{\circ}$ (perpendicular incidence) to $86^{\circ}$ (almost grazing incidence). Two series were manufactured: a general series modifying evaporation angle from $0^{\circ}$ to $80^{\circ}$ in $10^{\circ}$ steps and another one for high evaporation angles from $80^{\circ}$ to $86^{\circ}$ in $2^{\circ}$ steps, in which the liquid crystal alignment suffered the most significant variations. For each evaporation angle, corresponding cells with ITO electrodes were assembled and filled with liquid crystal and the pretilt angle was measured with respect to the perpendicular plane (Fig. 1).

\section{Results}

\section{1. $\mathrm{SiOx}$ and $\mathrm{SiO}_{2}$ morphological study}

\subsubsection{SiOx morphology}

A sweep of angles (increasing step in $10^{\circ}$ for each set of samples) was carried out to determine where the relevant morphological changes take place.

The acquired images correspond to evaporation angles of $10^{\circ}, 20^{\circ}, 30^{\circ}, 40^{\circ}, 50^{\circ}, 60^{\circ}, 70^{\circ}, 80^{\circ}, 82^{\circ}, 84^{\circ}, 85^{\circ}$ and $86^{\circ}$. Every angle was repeated at least 5 times, placing 8 substrates ( 4 cells) on each batch.

Structures from $20^{\circ}$ to $80^{\circ}$ are similar to each other; a uniform structure is observed in the whole sample. Observation of the samples became more difficult for this reason, surfaces being flat, homogenous and without uneven features, so finding a focus point turned out to be an extraordinary difficult task, especially when interesting features appeared only in high zoom $(\times 60000)$.

As seen in Fig. $2(\times 60000)$ surface morphology in this angle range does not show any recognizable polyhedral structure of the pseudo triangular SiOx particles. SiOx features are non-uniform rounded shaped particles, with no preferred orientation. Particles appear to be scattered all over the substrate, with diameters ranging from 100 to 250 $\mathrm{nm}$ approximately. A steady increase of droplet size is observed when evaporation angle increases, only for lower evaporation angles. When SiOx thickness is increased, an oblique columnar structure shows up [12].

From $50^{\circ}$ to $80^{\circ}$ surface structures show some variations. Particles become more defined, with sharper delineated boundaries. They also have a more rounded shape and their size appears to be larger than for lower angles. Near $80^{\circ}$ surface structure starts being less uniform having little holes or cracks between particles.

A sudden change in morphology occurred for angles greater than $80^{\circ}$. A more detailed study was done for samples with evaporation angles from $80^{\circ}$ to $86^{\circ}$, as its morphology showed a remarkable alteration: structure and particle size were different, and particle distribution highly differs. In this case surface was less uniform than the surface observed at lower evaporation angles, showing irregularities along the whole sample and a more detailed structure. The particle size changes, showing uniformity and diameters from $30 \mathrm{~nm}$ to $50 \mathrm{~nm}$.

In these cases, a progressive evolution of the surface structure was observed, changing from 'pebble dash-like' structure to a more granular spherulitic morphology. Evapo-

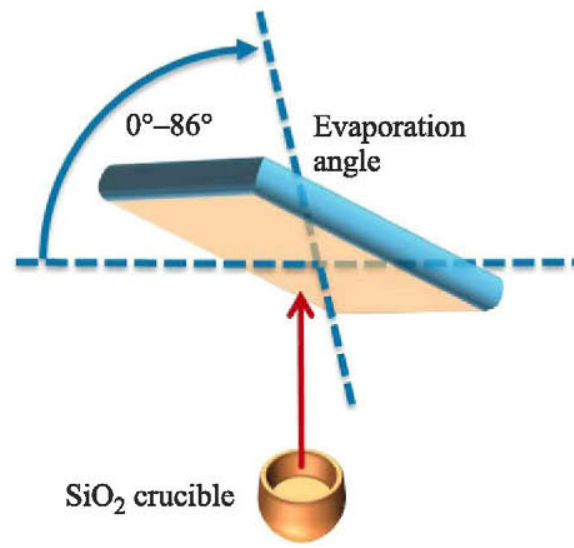

(a)

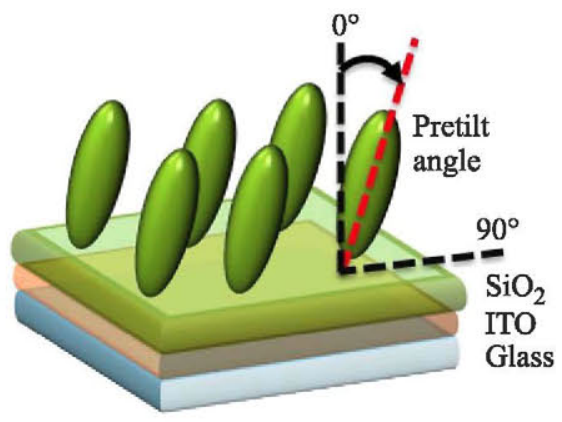

(b)

Fig. 1. Scheme illustrates the geometry for (a) the evaporation angle of the substrates and (b) the pretilt angle measured with respect to the perpendicular plane. 

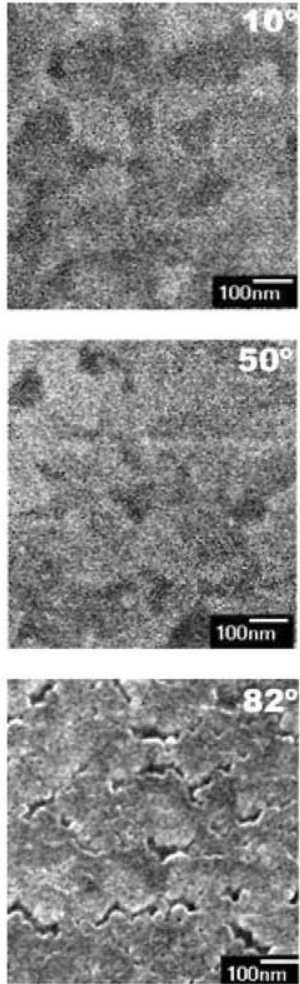
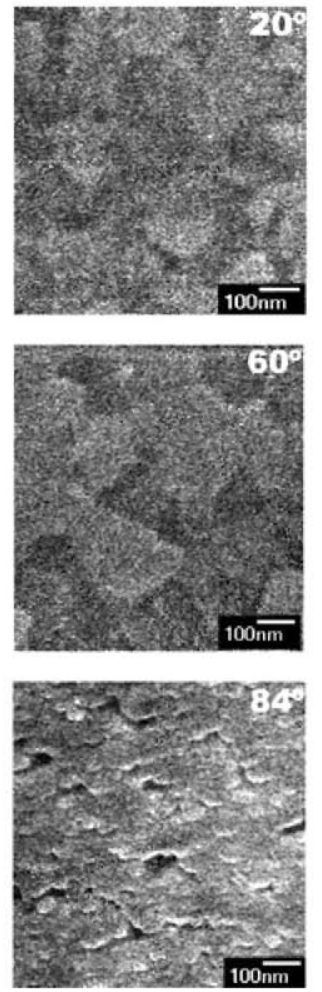
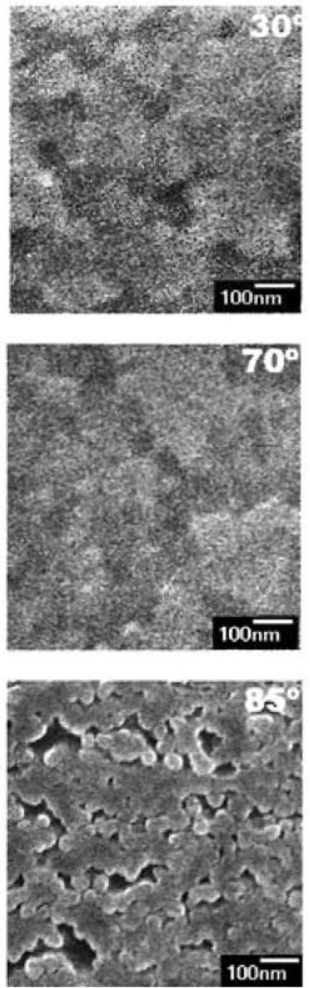
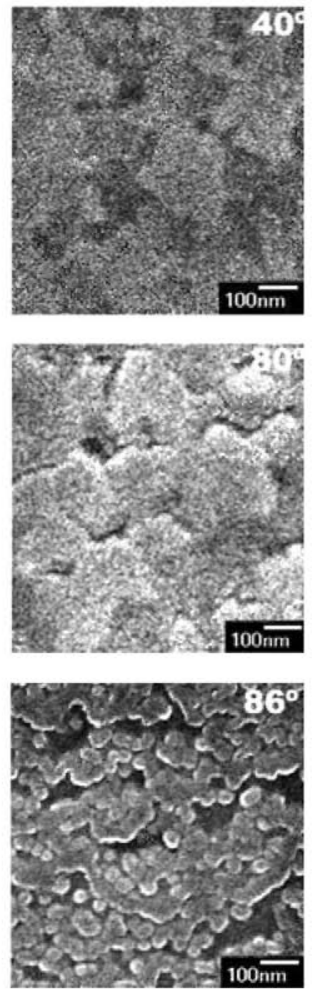

Fig. 2. Morphology structures of $\mathrm{SiOx}$ from $10^{\circ}$ to $86^{\circ}$.

rations for $82^{\circ}$ and $84^{\circ}$ show intermediate morphology states, coexisting both 'pebble dash' and spherulitic structures.

High angles, $85^{\circ}$ and $86^{\circ}$ show a spherulitic morphology, with sharper edges defined, smaller particles and presence of holes at high angles. 'Pebble dash-like' structures are no longer observed. Holes are triangular shaped, in accordance with a spherical stacking. Additionally, structures at higher angles are less compact, have bigger holes and spherical isolated particles are present. Alignment of particles and holes perpendicular to evaporation direction is observed. Thicker SiOx layers lead to an oblique columnar structure.

To summarize, when evaporation angle increases:

- There is a progressive evolution from 'pebble dash-like' structure to spherulitic structure;

- Particle size decreases from 150 to $50 \mathrm{~nm}$, approximately;

- Increasingly bigger holes between particles appear;

- Particle size is homogenous within the same substrate. This is more apparent at higher evaporation angles;

- Structures become less packed structures with bigger triangular shaped holes;

- The particle distribution order level increases, leading into a granulate morphology with a preferential structure direction.

\subsection{2. $\mathrm{SiO}_{2}$ morphology}

The same morphological study was carried out for $\mathrm{SiO}_{2}$ samples, the evaporation angles ranging from $10^{\circ}$ to $86^{\circ}$, as well. In general terms the observed morphology structures have similarities to $\mathrm{SiOx}$ structures but changes are more abrupt.

From $10^{\circ}$ to $65^{\circ}$ the morphology shows little resemblance with the 'pebble dash-like' morphology observed in $\mathrm{SiOx}$ at low evaporation angles as seen in Fig. 3 ( $\times 60000)$. These structures show little features and defined shapes and, in general, they are more compact, flat and uniform than their SiOx equivalent. It is worth mentioning that, under $40^{\circ}$, image acquisition in FESEM was very difficult due to the layers being featureless flat structures, images were not focused enough and, therefore, they are not shown in the image series.
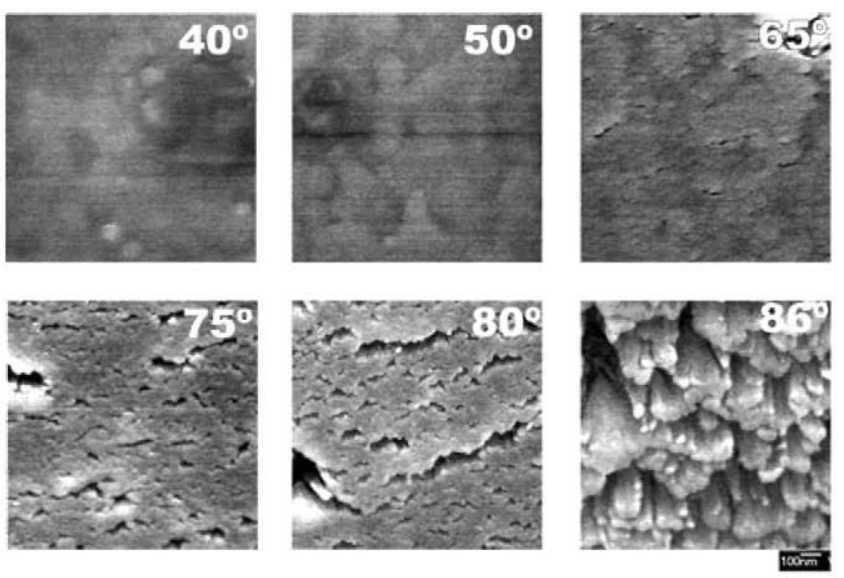

Fig. 3. Morphology structures of $\mathrm{SiO}_{2}$ from $40^{\circ}$ to $86^{\circ}$ evaporation angles. 
From $65^{\circ}$ upwards there is a morphologic change; less compact structures, holes and defined particles show up. This is an interesting fact because $65^{\circ}$ is the angle in which pretilt angle starts to change. $75^{\circ}$ and $80^{\circ}$ showed a more granular, less compact structure. Particles grow into smaller grains, its shape is more defined and triangular holes become bigger and more frequent. In this stage some tendency to columnar morphology can be observed; no spherulitic structure (as in $\mathrm{SiOx}$ ) but a pseudo-columnar structure was identified. At $86^{\circ}$ the morphology becomes less packed. A columnar structure is observed: columns are prism shaped and some triangular shaped bigger particles are seen [13, 14]. There is a preferential growth direction, corresponding to the evaporation direction.

When evaporation angle increases:

- Morphology structure evolves from a compact pseudo-droplet structure into a columnar structure;

- Particle size decreases from 200 to $100 \mathrm{~nm}$, approximately;

- Triangular shaped holes appear at higher evaporation angles;

- Particle size is more homogenous within the same substrate at higher evaporation angles;

- Structures become less packed with bigger triangular shaped holes;

- At higher angles a columnar structure with prism-shaped columns is observed;

- The particle distribution order level increases growing with a preferential structure direction;

- In general, morphology changes are more marked than in $\mathrm{SiOx}$.

\subsection{Electrooptic characterization}

Measurements carried out for VAN cells were focused on a comparative study of response times and contrast ratios depending on the different evaporation angles. Moreover, a grayscale study was implemented to observe differences in liquid crystal behaviour. All experiments were performed using a commercial negative nematic dielectric anisotropic liquid crystal mixture: MLC-6608 (Merck).

Electrooptic properties of VAN cells depend directly of manufacturing factors, which control, among other characteristics, the pretilt angle of the liquid crystal. Pretilt angle together with cell thickness and anchoring energy, are the most relevant factors to set properties like response time and contrast ratio.

While measuring response times, an anomalous behaviour of the liquid crystal was detected. This phenomenon, called backflow effect, is explained below.

\subsubsection{Liquid crystal alignment}

Two series of liquid crystal cells were manufactured with $\mathrm{SiOx}$ and $\mathrm{SiO}_{2}$ as alignment layers, changing evaporation angle was increased by $10^{\circ}$ steps in angle ranges where little variations had been detected in previously described microscopy batches. Otherwise $2^{\circ}$ steps were used. The ITO monopixel electrode consisted of a $1 \mathrm{~cm}^{2}$ square with an electrode for external driving (normal field). Cells were assembled in an antiparallel manner. Overall, about 90 cells were manufactured for each batch of this experiment.

The alignment of the liquid crystal was assessed between crossed polarizers, placing the liquid crystal cell evaporation direction at $45^{\circ}$ with respect to the polarizers. The optical axis of the liquid crystal corresponds to the evaporation direction. The transmission with this rotation depends on the birefringence of the cell, and, thus on the pretilt. In zero pretilt cells the birefringence is zero, therefore the cell appears dark. The transmitted intensity increases as the birefringence increases with pretilt. The pretilt angle was obtained for all cases.

The main conclusions are:

- In $\mathrm{SiOx}$, evaporation angles lower than $50^{\circ}$ do not align the liquid crystal uniformly; from $50^{\circ}$ to $80^{\circ}$ liquid crystal is aligned homeotropically;

- In $\mathrm{SiO}_{2}$, evaporation angles from $0^{\circ}$ to $80^{\circ}$ produce homeotropic alignment of the liquid crystal;

- In both cases, liquid crystal alignment for angles higher than $80^{\circ}$ is nearly homogenous, with a very high pretilt.

\section{a. Alignment in $\mathrm{SiOx}$}

In Fig. 4, several cells between crossed polarizers can be observed. Samples with evaporation angles from $20^{\circ}$ to $50^{\circ}$ present mostly a focal conic alignment with no clearly determined orientation direction. Alignment in samples with

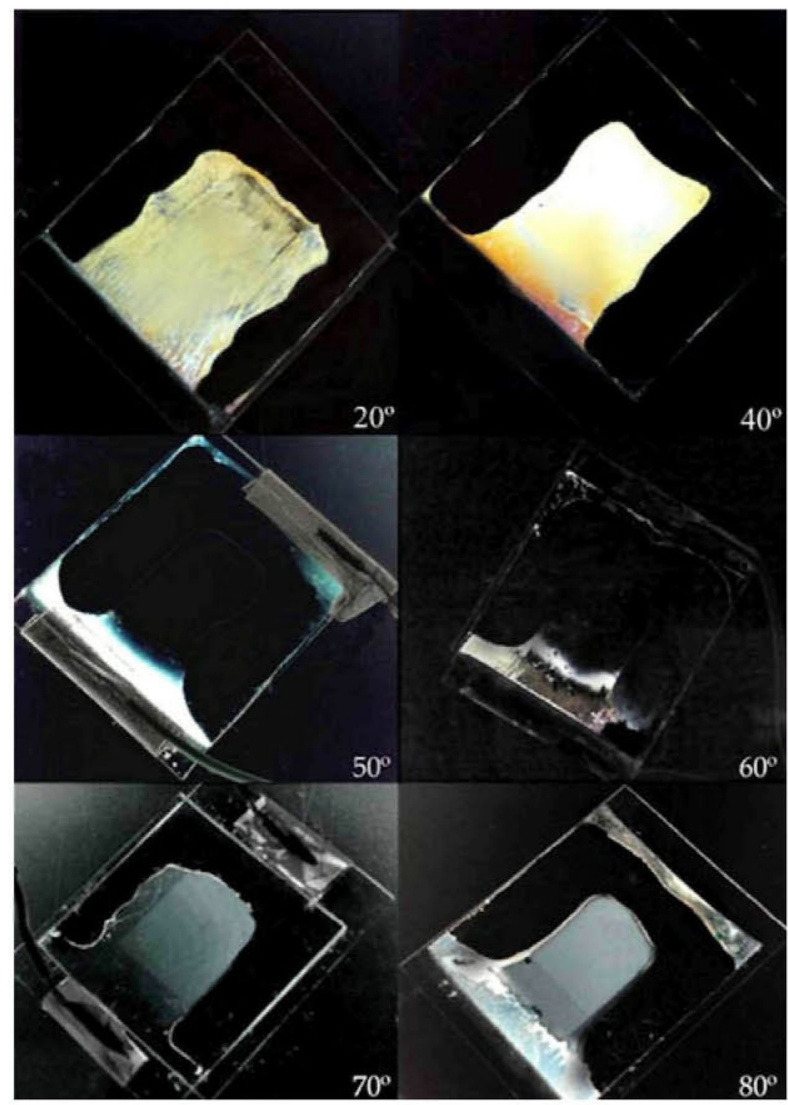

Fig. 4. VAN liquid crystal test cells at different SiOx evaporation angles as seen between crossed polarizers. 
evaporations from $50^{\circ}$ to $80^{\circ}$ presents the most interesting results.

It is worth noticing that, as mentioned above, only minute variations in the $\mathrm{SiOx}$ surface morphology are appreciated when comparing surfaces in the $20^{\circ}-50^{\circ}$ range and surfaces in the $50^{\circ}-80^{\circ}$. These minute variations scarcely visible at very high zoom give rise, however, to a dramatic change in the liquid crystal director orientation.

$50^{\circ}$ and $60^{\circ}$ cells shows no transmission (dark), therefore pretilt is null or near null, and cell will show a good contrast ratio. Alignment obtained for $70^{\circ}$ upwards cells differs. Homeotropic alignment is achieved as well, but a certain transmission in the cell can be seen. $80^{\circ}$ cells exhibit the highest transmission; pretilt angle increases considerably (reaching values as high as $25^{\circ}$ ), from $80^{\circ}$ to $86^{\circ}$ showed interesting variations. As mentioned before, $\mathrm{SiOx}$ morphology for angles greater than $80^{\circ}$ changes drastically compared to lower angles. Liquid crystal alignment manifests a variation in the same way. For high angles, pretilt angle is so high that alignment cannot be considered homeotropic anymore. The contrast ratio is consequently compromised.

\section{b. Alignment in $\mathrm{SiO}_{2}$}

Liquid crystal alignment in $\mathrm{SiO}_{2}$ is similar to that on $\mathrm{SiOx}$. The main differences are found in the lower angle range.

In $\mathrm{SiO}_{2}$ liquid crystal alignment is homeotropic from $0^{\circ}$ to $80^{\circ}$, with pretilt variations as the angle increases. Cells observed between crossed polarizers showed a complete dark state (zero pretilt) in the $0^{\circ}-60^{\circ}$ range (Fig. 5). Pretilt measured in this range is zero or near zero. As in $\mathrm{SiOx}$ cells, when evaporation angle increases, from $70^{\circ}$ upwards, cells start to show some transmission and less contrast ratio. Pretilt values are around $15^{\circ}$. From $80^{\circ}$ to $86^{\circ}$ transmission and pretilt increase considerably. Evaporation angle of $86^{\circ}$ produces an almost homogeneous alignment, molecules having a pretilt close to $55^{\circ}$. Contrast in these cells is very low, and switching is barely noticeable. Note that switching would intend to bring the molecules to any position within the substrates plane. Since the molecules are almost positioned on this plane in steady state, no reorientation is required for the liquid crystal to switch.

A scheme of the liquid crystal alignment depending on the evaporation angle of both $\mathrm{SiOx}$ and $\mathrm{SiO}_{2}$ is shown in Fig. 6.
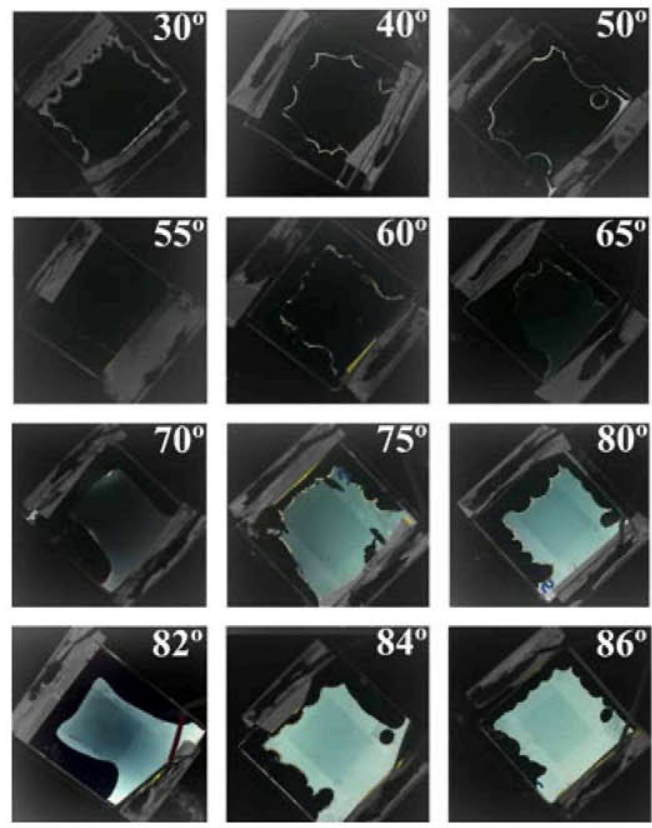

Fig. 5. VAN liquid crystal test cells at different $\mathrm{SiO}_{2}$ evaporation angles as seen between crossed polarizers.

\subsubsection{Backflow effect}

Rise time of any liquid crystal display is usually shorter as the applied voltage increases, since liquid crystal molecules switch faster at higher voltages. However, in a number of manufactured cells the opposite behaviour was observed: the response time of cells having null or near null pretilt angle increases above a certain voltage and the rise time becomes progressively longer. However, fall times were not affected. This effect remarkably alters the measurement of response times; it is attributed to the so-called backflow effect [15].

Above certain voltages, a fast rearrangement (turbulences) of the liquid crystal molecules is induced followed by a slow reorientation that induces the molecules to switch slowly increasing the overall rise time. From the practical point of view, cells showing backflow have a limited voltage range. Above that range, response time is so long that cells become useless for most applications. During this study, it was found that the presence or absence of backflow depends strongly on the pretilt and on the cell thickness:

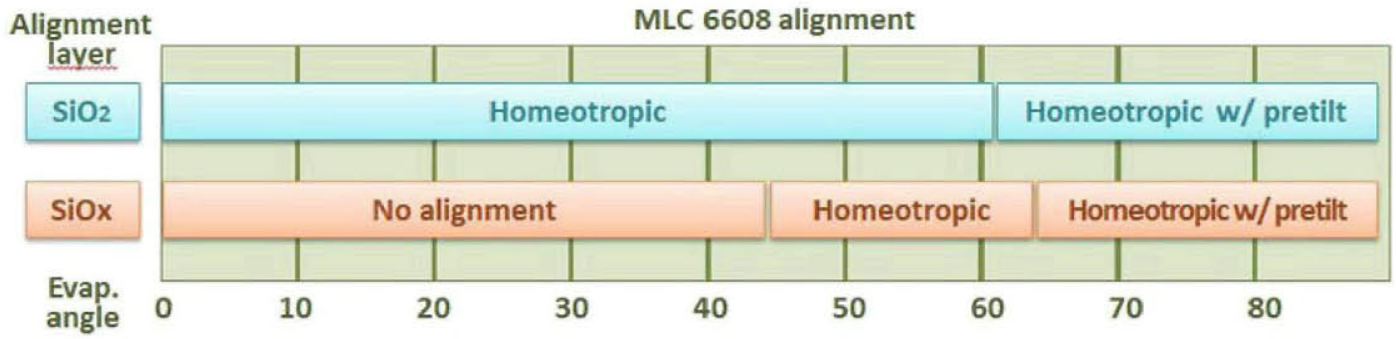

Fig. 6. Liquid crystal alignment vs. evaporation angle. 
- Blackflow arises only in cells with null or very low pretilt and, thus the issue is important since these cells are usually the most interesting in applications because of their high contrast.

- Cells without backflow effect can be obtained by reducing cell thickness while keeping constant the remaining manufacturing parameters. When thickness is reduced, backflow either disappears or can be only detected at higher voltages.

\subsubsection{Electrooptic measurements}

\section{a. Electrooptics in $\mathrm{SiOx}$}

An electrooptic characterization was made for all cells ranging from $50^{\circ}$ to $85^{\circ}$. Figure 7 shows grayscale and dynamic response for $50^{\circ}, 60^{\circ}, 70^{\circ}$ and $80^{\circ}$ samples. Cells were excited in all cases with a pulsed AC signal of $1 \mathrm{kHz}$ and variable amplitude. The cell threshold was about $2.5 \mathrm{~V}$ in all cases, whereas the saturation voltage was about $10 \mathrm{~V}$.

$50^{\circ}$ and $60^{\circ}$ cells, having very low pretilt, exhibited backflow when applying voltages greater than $6 \mathrm{~V}$, showing a double-peaked transmission profile and slow response times. For example, the rise time for $10 \mathrm{~V}$ was $20 \mathrm{~ms}$. Nevertheless, in a working range up to $6 \mathrm{~V}, 50^{\circ}$ and $60^{\circ}$ displays show very good response time $(3 \mathrm{~ms})$ and contrast ratios above 500. These are values compatible with high-end video rate applications such as direct-view TV [16].

In $70^{\circ}$ displays, backflow effect did not show up. As pretilt angle increases, switching becomes easier, so turbulences do not appear and backflow effect is avoided. Response times are fast in the whole voltage range (3-4 $\mathrm{ms}$ ), faster than for $50^{\circ}$ or $60^{\circ}$. Contrast, however, decreases as a result of the birefringence in the off-state induced by the pretilt angle $(<100)$.
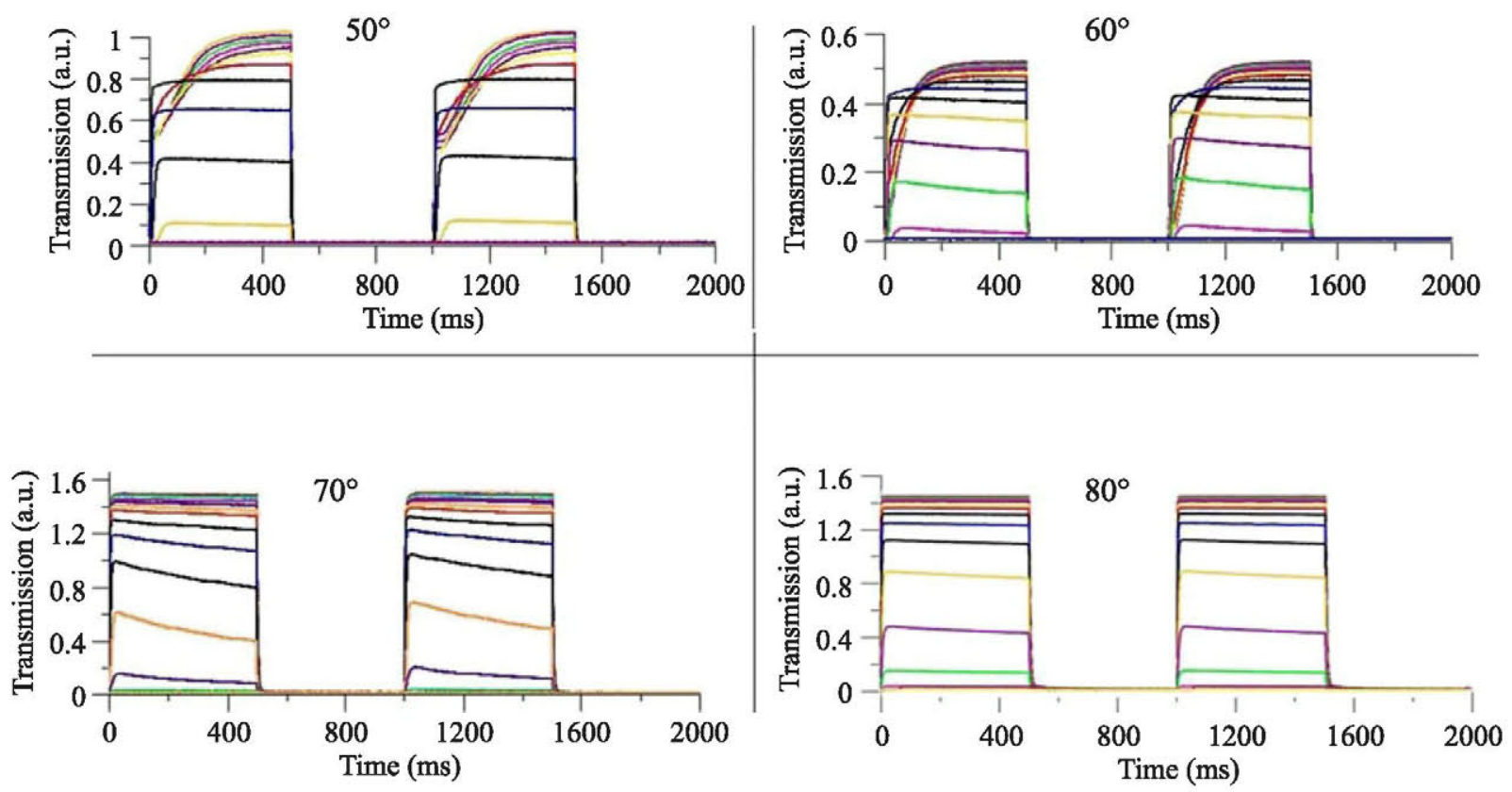

Fig. 7. Dynamic response for $50^{\circ}, 60^{\circ}, 70^{\circ}$ and $80^{\circ}$ evaporation of $\mathrm{SiOx}$. $80^{\circ}$ cells response is similar to that of $70^{\circ}$. In these cells, however, pretilt is very high (about $26^{\circ}$ ), making the off-state highly transmissive. Backflow could not be found in these displays either and response times were the fastest due to high pretilt. Fall times increase as pretilt increases, opposite to rise time. This behaviour can be explained by differences in the elastic energy. The higher the pretilt is, the smaller the elastic energy between on and off state becomes, consequently, for that reason longer fall times are expected.

\section{b. Electrooptics in $\mathrm{SiO}_{2}$}

$\mathrm{SiO}_{2}$ cell behaviour is similar to the $\mathrm{SiOx}$ cells. From $0^{\circ}$ to $60^{\circ}$ a strong backflow effect can be seen, due to the pure homeotropic alignment present in these cells. Rise times are high, varying from a hundred to tens of milliseconds at saturation voltage. (Fig. 8). In a number of cases, some cells with zero pretilt showed a variable backflow effect in time and, thus a variable switching time. This variable rise time is consistent with how backflow effect works in pure homeotropic alignment. As turbulences are involved in the liquid crystal, stochastic and chaotic flows are present until molecules are able to reorient towards the applied field.

From $65^{\circ}$ upwards backflow effect disappears and rise times are always under $10 \mathrm{~ms}$, even under $1 \mathrm{~ms}$ for the highest evaporation angles $\left(80^{\circ}-86^{\circ}\right)$. As in $\mathrm{SiOx}$ cells, when pretilt is high, fall time increases considerably.

In general terms the absence of backflow and the excellent response time should make displays in the range of $70^{\circ}-80^{\circ}$ the best choice for standard applications. Nevertheless, contrast is poor, thus precluding their use in display applications. This is not the case, however, for photonic applications where variable phase delays of VANs are used. In this case, the only drawback of high pretilt is that the range of delays generated by a given liquid crystal material and cell thickness is somewhat reduced. This small disad- 


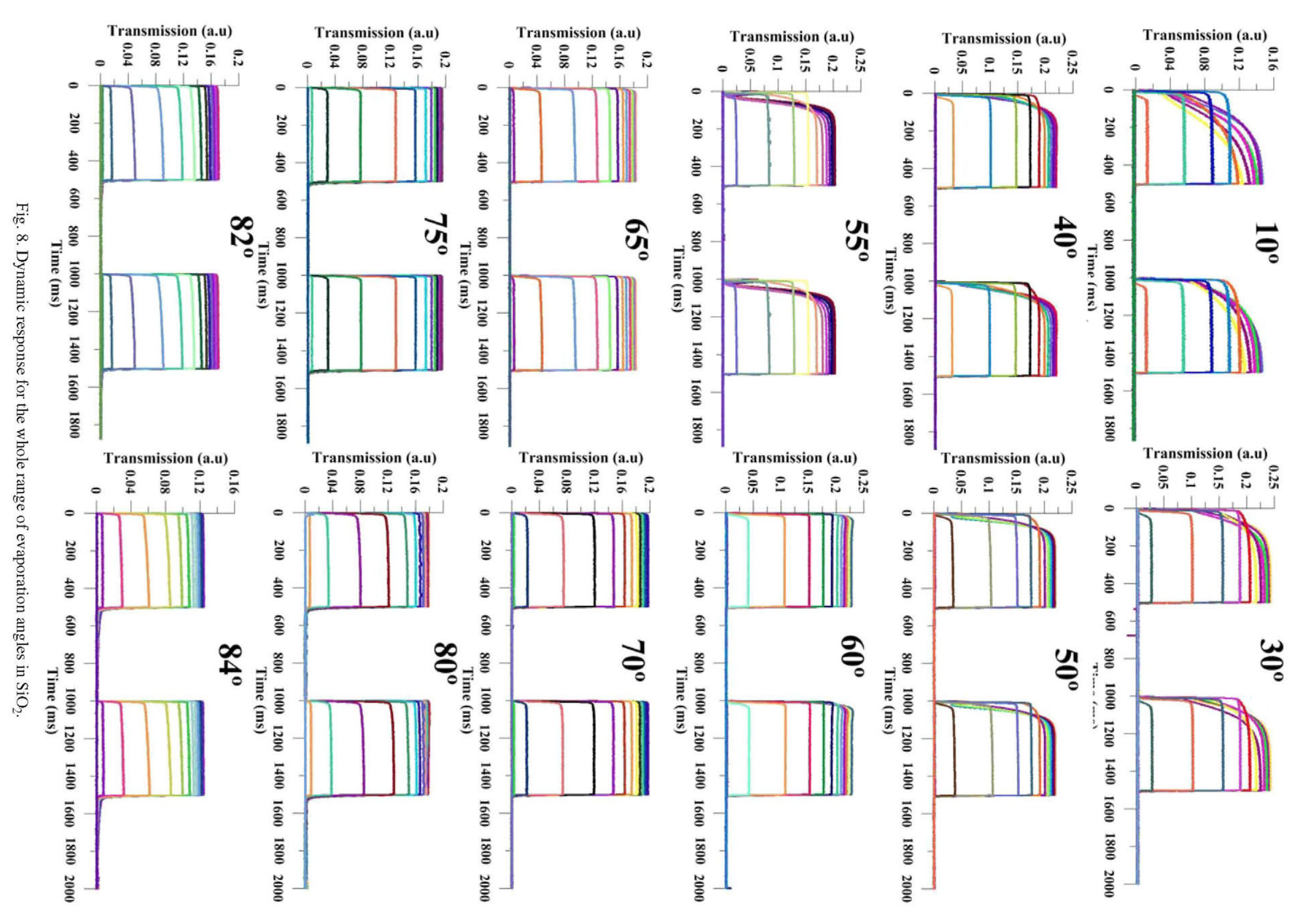


vantage is superseded in many applications by the excellent dynamic behaviour of high pretilt cells.

\subsubsection{Response time measurements}

A series of cells were measured, using a $1 \mathrm{kHz}$ sin wave modulated with a $1 \mathrm{~Hz}$ square wave. Response time is measured as the time the liquid crystal cell takes to switch from $10 \%$ to $90 \%$ transmission. Both switch on and switch off (rise and fall) times were measured. Mean rise and fall times for the different evaporation angles are compared in Table 1 for $\mathrm{SiOx}$ and $\mathrm{SiO}_{2}$ cells, respectively. In general, given an evaporation angle, $\mathrm{SiO}_{2}$ layer produces faster response times. This is consequent with the pretilt measurements: given an evaporation angle, the obtained pretilt is higher when using $\mathrm{SiO}_{2}$ than when using $\mathrm{SiOx}$ as alignment layer.

Rise times decrease when increasing pretilt angle; the higher the pretilt angle is, the easier the switching becomes

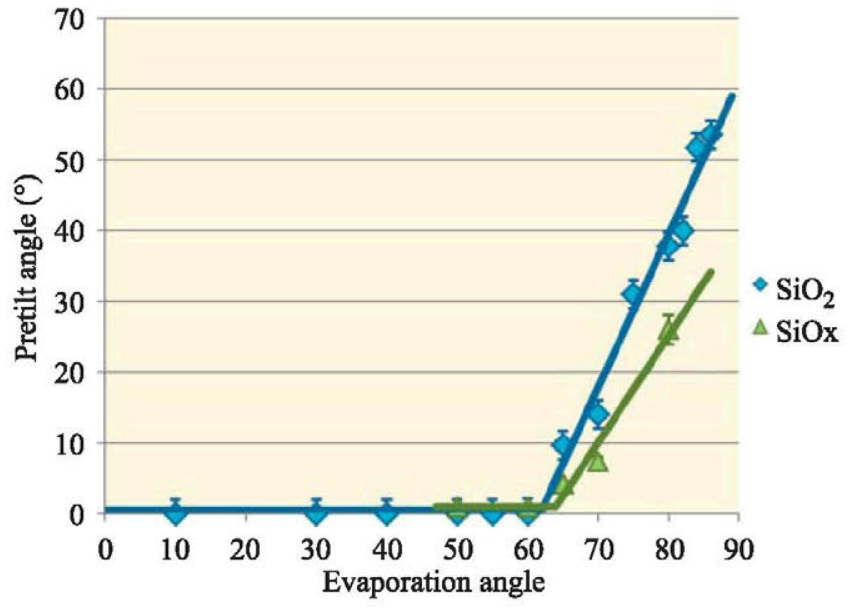

Fig. 9. Pretilt vs. evaporation angle in $\mathrm{SiO}_{2}$ cells (blue) and $\mathrm{SiOx}$ cells (green).

Table 1. Mean response times for $\mathrm{SiOx}$ and $\mathrm{SiO}_{2}$ VAN cells.

\begin{tabular}{lccccccccccccc}
\hline Evap. angle & $10^{\circ}$ & $30^{\circ}$ & $40^{\circ}$ & $50^{\circ}$ & $55^{\circ}$ & $60^{\circ}$ & $65^{\circ}$ & $70^{\circ}$ & $75^{\circ}$ & $80^{\circ}$ & $82^{\circ}$ & $84^{\circ}$ & $86^{\circ}$ \\
\hline SiOx Rise time (ms) & - & - & - & 153 & - & 94 & - & 8.0 & - & 1.3 & - & - & 0.6 \\
$\mathrm{SiO}_{2}$ Rise time (ms) & 295 & 71 & 68 & 63 & 45 & 31 & 11 & 4.9 & 0.70 & 0.42 & 0.38 & 0.41 & 0.45 \\
$\mathrm{SiOx}$ Fall time (ms) & - & - & - & 5.6 & - & 4.8 & - & 6.6 & - & 7.5 & - & - & 15 \\
$\mathrm{SiO}_{2}$ Fall time (ms) & 5.0 & 5.7 & 5.4 & 5.8 & 5.2 & 4.7 & 5.9 & 5.8 & 9.9 & 11 & 7.2 & 9.9 & 10 \\
\hline
\end{tabular}

for liquid crystal. The data collected for cells with zero or near zero pretilt were slightly scattered; very little differences of pretilt in pure homeotropic alignment (having $0^{\circ}$ or $1^{\circ}$ pretilt angle) makes a significant difference in terms of switching time.

Fall times increase as pretilt angle increases; liquid crystal molecules are less attached to the surface. Anchoring energy of the liquid crystal molecules decreases when pretilt is high and, in consequence, it takes them longer to come back to the relaxed state. A good balance between rise and fall times can be set up for photonic applications depending on the evaporation angle.

\subsubsection{Pretilt and contrast measurements}

\section{a. Pretilt measurements}

Pretilt angle was measured for all $\mathrm{SiOx}$ and $\mathrm{SiO}_{2}$ cells, see Table 2.

The pretilt angle distribution can be measured by different methods [17]. The technique in this case consisted in measuring transmission changes in a cell (always in off-state) when incident light angle is modified.

The cells were mounted in a rotatory stage aligned with a $\mathrm{He}-\mathrm{Ne}$ laser with a maximum output peak of $5 \mathrm{~mW}$ at
$543 \mathrm{~nm}$, a polarizer, a photodetector and a Stanford Research Systems DS345 waveform generator. Transmission levels are analysed in a Tektronik TDS 714L digital oscilloscope. These measurements are less precise for measuring low pretilt angles $\left(\approx 1^{\circ}-2^{\circ}\right)$ but this method was used for comparative purposes only. More accurate measurements were done using an ellipsometric technique and were presented in Ref. 18.

Given a certain evaporation angle (from $65^{\circ}$ upwards) pretilt is higher in $\mathrm{SiO}_{2}$ cells than in $\mathrm{SiOx}$ cells. Additionally, pretilt angle increases faster in $\mathrm{SiO}_{2}$ than in $\mathrm{SiOx}$ cells when increasing evaporation angle (Fig. 9). This is consequent with the morphology structure changes explained in previous sections.

\section{b. Contrast ratio}

Contrast ratio was measured for a set of $\mathrm{SiO}_{2}$ cells, in the same setup used for measuring pretilt angles. Changes of transmission were measured in all cells in OFF state (minimum transmission) and $\mathrm{ON}$ state (maximum transmission).

Contrast ratio values were obtained from the transmission measurements and normalized; the mean contrast ratio values are plotted in Fig. 10.

Table 2. Mean pretilt values for $\mathrm{SiOx}$ and $\mathrm{SiO}_{2}$ VAN cells.

\begin{tabular}{|c|c|c|c|c|c|c|c|c|c|c|c|c|c|}
\hline Evap. angle & $10^{\circ}$ & $30^{\circ}$ & $40^{\circ}$ & $50^{\circ}$ & $55^{\circ}$ & $60^{\circ}$ & $65^{\circ}$ & $70^{\circ}$ & $75^{\circ}$ & $80^{\circ}$ & $82^{\circ}$ & $84^{\circ}$ & $86^{\circ}$ \\
\hline SiOx pretilt $\left( \pm 1^{\circ}\right)$ & - & - & - & $0^{\circ}$ & $0^{\circ}$ & $0^{\circ}$ & $5^{\circ}$ & $8^{\circ}$ & - & $25^{\circ}$ & - & - & - \\
\hline $\mathrm{SiO}_{2}$ pretilt $\left( \pm 1^{\circ}\right)$ & $0^{\circ}$ & $0^{\circ}$ & $0^{\circ}$ & $0^{\circ}$ & $0^{\circ}$ & $0^{\circ}$ & $9^{\circ}$ & $14^{\circ}$ & $31^{\circ}$ & $38^{\circ}$ & $40^{\circ}$ & $51^{\circ}$ & $53^{\circ}$ \\
\hline
\end{tabular}




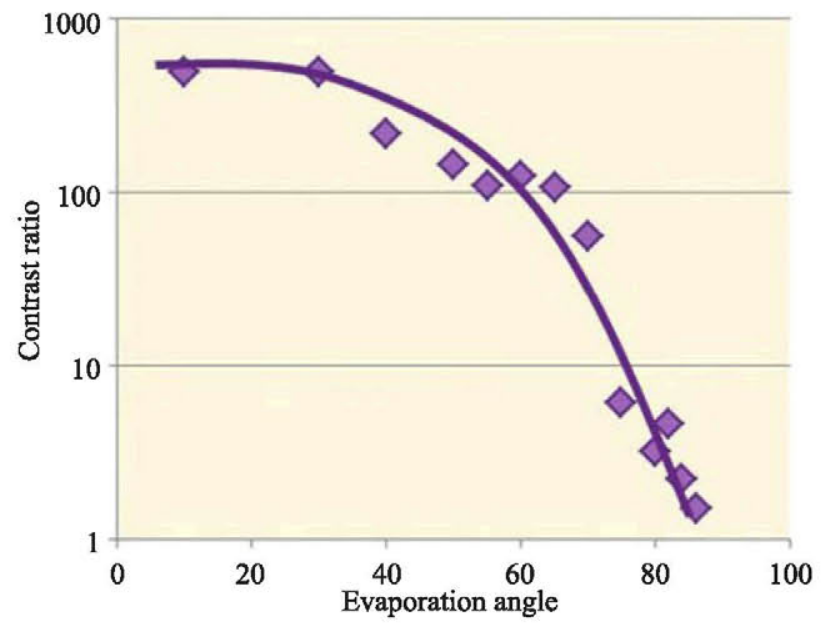

Fig. 10. Contrast ratio vs. evaporation angle in $\mathrm{SiO}_{2}$ cells.

\section{Conclusions}

Results prove that both $\mathrm{SiOx}$ and $\mathrm{SiO}_{2}$ as aligning surfaces for VAN liquid crystals may generate a full range of pretilt angles that can eventually be optimized, depending on the requirements of the specific application. In principle, display-related applications require very low pretilts, hence low evaporation angles, so that contrast is maximized.

Our group has proposed a method to achieve a trade-off between contrast ratio and response time. The method is based on the use of asymmetric alignment layers. Combining glass plates evaporated at different angles, improvements in contrast ratio without jeopardizing the response time have been found [19].

Contrast requirements shall be met at its minimum for the response time to be improved as much as possible. For non-display applications, silicon oxides are a very good choice for certain devices, like aerospace applications, due to the inherent inertness of inorganic alignment layers [20]. In these cases, higher pretilt angles would probably be chosen to minimize the response time. The slightly lower phase delay range achieved in these cells should not be relevant for the device performance in most cases.

\section{Acknowledgements}

Authors are grateful for the financial support from Spanish Ministerio de Ciencia e Innovación, project TEC2008-00147 (ALCOD) and Madrid Regional Program FACTOTEM S2009/ESP1781.

\section{References}

1. J.L. Janning, "Thin film surface orientation for liquid crystals", Appl. Phys. Lett. 21 (4), 173-175 (1972).

2. E. Otón, S. López-Andrés, N. Bennis, X. Quintana, J.M. Otón and C. Carrasco, "Electrooptic and surface study of vertically aligned nematics with induced pretilt", IEEE $J$. Disp. Technol. 6 (7), 263-268 (2008).

3. S. Choi, J. Hwang, S. Kim, B. Oh, J. Myoung and D. Seo, "Liquid Crystal Alignment and Electrooptical Characteris- tics of Vertical Alignment Liquid Crystal Display on SiOx Thin Film Obliquely Deposited by Sputtering", Jpn. J. Appl. Phys., 45, 1280-1282 (2006).

4. S.J. Sung, K.J. Yang, D.H. Kim, Y.S. Do, J.K. Kang and B.D. Choi, "The electro-optical behavior of liquid crystal molecules on the surface of $\mathrm{SiO}_{2}$ inorganic thin films", $J$. Nanosci. Nanotechnol., 9 (12), 6938-42 (2009).

5. C. Chen, P.J. Bos, J. Kim, Q. Li and J.E. Anderson, "Improved Liquid Crystals for Vertical Alignment Applications", J. Appl. Phys., 99, 123523 (2006).

6. P.K. Son, J.H. Park, S.S. Cha, J.C. Kim, T.H. Yoon, "Vertical alignment of liquid crystal on a-SiOx thin film using the ion beam exposure", Appl. Phys. Lett., 88, 263512 (2006).

7. T. Shimamura, H. Okada and H. Onnagawa. "Vertically Aligned Nematic Liquid Crystal Display Using Dual-Interference Exposure Method", Jpn. J. Appl. Phys., 43, 256-260 (2004).

8. K.Y. Yu, C.R. Lee, C.H. Lin and C.T. Kuo, "Controllable pretilt angle of liquid crystals with the formation of microgrooves", J. Phys. D: Appl. Phys. 46045102 (2013).

9. P.J. Martin, A. Bendavid, C. Comte, H. Miyata, Y. Asao, Y. Ishida, A. Sakai, "Alignment and switching behaviors of liquid crystal on a-SiOx thin films deposited by a filtered cathodic arc process", Appl. Phys. Lett., 91, 063516 (2007).

10. B. Jerome, P. Pieranski, M. Boix, "Bistable anchoring of nematics on SiO films", Europhys. Lett., 5, 693 (1988).

11. M. Monkade, M. Boix, G. Durand, "Order Electricity and Oblique Nematic Orientation on Rough Solid Surfaces", Europhys. Lett., 5, 697 (1988).

12. S. Kondoh, "Ultra high-resolution FLC display for PDA", Display 25, 31-36 (2004).

13. H. Yokoyama, S. Kobayashi, H. Kamei, "Role of surface adsorption in the surface-induced alignment of nematic liquid crystals on evaporated SiO films", J.Appl. Phys. 56, 2645 (1984).

14. H.A. van Sprang, R.G. Aartsen, "The temperature dependence of liquid-crystal tilt angles", J.Appl. Phys. 56, 251 (1984).

15. P.J.M. Vanbrabant, N. Dessaud and J.F. Strömer, "Temperature influence on the dynamics of vertically aligned liquid crystal displays", Appl. Phys. Lett. 92, 091101 (2008).

16. J.J. Lyu, J. Sohn, H.Y. Kim, S.H. Lee, "Recent trends on patterned vertical alignment (PVA) and fringe-field switching (FFS) liquid crystal displays for liquid crystal television applications". J. Disp. Technolog., 3 (4), 404-412, (2007).

17. X. Quintana, M.A. Geday, B. Cerrolaza, D. Pérez-Medialdea and J.M. Otón, "Measurement of pretilt angle and cell gap in reflective vertically aligned nematics", in Proc. 6th IEEE Spanish Conference on Electron Devices, pp. 277-280, El Escorial, Spain, 2007.

18. N. Bennis, E. Otón, A. Marino, V. Tkachenko and X. Quintana, "Ellipsometric study of VANs with silicon oxides as alignment surfaces" in 20th Conference on Liquid Crystals, Mikołajki, Poland, 2013.

19. A. Marino, E. Santamato, N. Bennis, X. Quintana, J.M. Otón, V. Tkachenko and G. Abbate, "Ellipsometric study of vertically aligned nematic liquid crystals" App. Phys. Lett. 94, 013508 (2009).

20. M.A. Geday, X. Quintana, E. Otón, B. Cerrolaza, D. Lopez, F. Garcia de Quiros, I. Manolis and A. Short, "Development of liquid crystal based adaptive optical elements for space applications", Proc. Intl. Conf. on Space Optics ICSO 2010, Rhodes, Greece, 2010. 